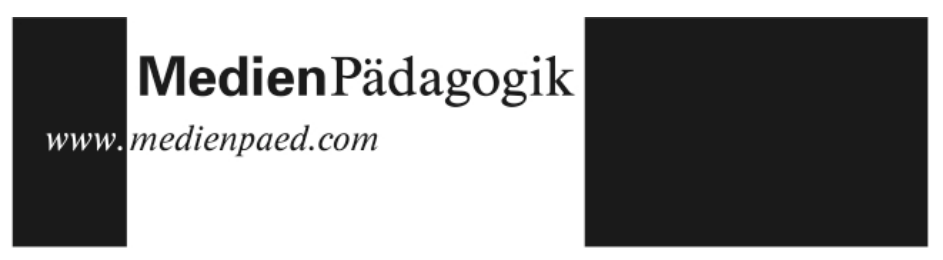

Rezensionen

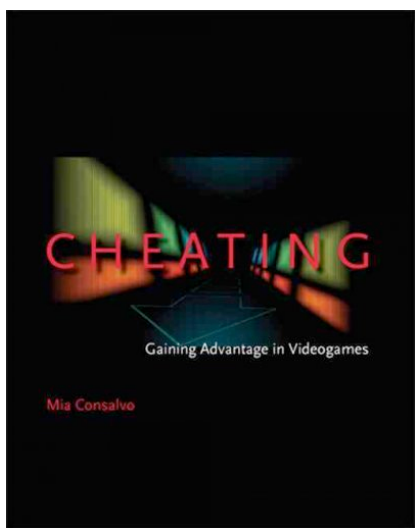

Mia Consalvo

Cheating. Gaining Advantage in Videogames.

Cambridge: MIT Press, Juli 2007, 232 Seiten

Stuttgart: Metzler, 2000. 667 Seiten

ISBN-10: 0262-03365-8

ISBN-13: 987-0-262-03365-7

Taschenbuch, $\$ 38,00 / £ 28$.

\title{
Cheating als Mehrwert - Kultur, Ökonomie, Macht
}

Die Game Studies, ein nach wie vor junges und vorwiegend interdisziplinär angelegte Forschungsfeld, erfahren mit dieser Monografie einen überaus leicht lesbaren und zugänglichen Beitrag, der technische, ökonomische, soziale und kulturelle Implikationen eines Phänomens näher beleuchtet, das für Computerspielerinnen und -spieler allgegenwärtig ist: Cheating. Die Übersetzung des Wortes cheating mit falsch spielen, mogeln oder schummeln verweist auf den ersten Blick auf moralisch zweifelhaftes Spielhandeln und ethische Fragen, die sich an das junge Medium Computerspiele stellen. Consalvo erforscht dieses Phänomen mit Rücksicht auf Wechselwirkungen und Interdependenzen zwischen den Spielerinnen und Spielern, den von innen präferierten Spielen, der Spieleindustrie, Spielemagazinen und Spielerforen im Internet, sowie technischer Modifikationen von Spielen wie Cheatcodes, Hacks oder Mods.

Mia Consalvo, die gegenwärtig als Visiting Associate Professor am MIT in Boston arbeitet, zieht zwei theoretische Konstrukte heran, um die Bedeutung des Cheating theoretisch fassbar zu machen: Zum einen entwickelt sie in Anlehnung an Pierre Bourdieus Konzept des kulturellen Kapitals den Begriff des gaming capital. Damit sucht sie zu veranschaulichen, welche auf Wissen, technischem Können und Spielerfahrung basierenden Austauschverhältnisse zwischen Spielenden und den sie umgebenden ökonomischen, sozialen und kulturellen Systemen entstehen. Zum anderen beschreibt sie unter Rekurs auf das Konzept des Paratexts, das der französische Literaturwissenschaftler und Narratologe Gérard Genette entwickelt hat, welche Rolle der breiten Spieleindustrie durch die Produktion von Spielen als Primärtext und Spielemagazinen, Game Guides, technischen Modifikationen 
von Spielen oder Onlineforen als Sekundärtext (Paratext) bei der Formung von gaming capital zukommt. Consalvo zeigt auf, dass diese Paratextindustrie in der konvergenten Medienwelt nicht nur eng mit der Kernindustrie verbunden ist, sondern gleichsam einen großen Beitrag zur Entwicklung der gegenwärtigen Computerspielkultur geleistet hat und leistet. Sie erläutert wie Paratext Einfluss darauf nimmt, wie Spieler spielen, über Spiele denken und sprechen, was ein Spiel zu einem guten Spiel macht, welche Handlungen aus moralischer Perspektive für Spielerinnen und Spieler akzeptabel sind und welche Sanktionen möglicherweise auf Fehlverhalten folgen.

Im ersten Teil des Buches wird ein kulturgeschichtlicher Überblick über die Entwicklung des Cheating in Computerspielen - von ersten dekorativen und funktionalen Code-Elementen in Spielen, über Spielanleitungen und Walkthroughs, externe Modifikationen und Chips bis hin zum Kauf von Items oder externen Dienstleitungen - und die Bedeutung der sich entwickelnden Paratextindustrie gegeben. Consalvo verdeutlicht hier, wie die Paratextindustrie die Entwicklung von "gaming capital» bei Spielerinnen und Spielern moderiert und welche Machtfragen dadurch aufgeworfen werden. Exemplifiziert werden diese Aspekte unterem anderem im Hinblick auf die Deutungshoheit darüber, wie Spiele zu spielen sind, welche Handlungen hier angemessen sind und in welchem Rahmen die Anwendung von Cheats und Modifikationen im Rahmen der Spielregeln als legitim gelten. Dass es sich dabei um Machtfragen handelt, wird auch bei juristischen Auseinandersetzungen über die Legitimität von Game Guides oder Mod Chips deutlich. Cheats gestatten es dem Spieler viel stärker, das Spiel mit seinen Regeln und Anforderungen an den Spieler selbst zu gestalten. Dieser Gewinn an Handlungsfreiheit begrenzt die Deutungsmacht der Hersteller (z.B. in welcher Reihenfolge welche Levels zu durchlaufen sind, ob man im Sinne von «gaming-the-game» dem Spiel andere Regeln zugrunde legt oder dieses für andere Tätigkeiten öffnet (z.B. für die Produktion von Machinimafilmen zweckentfremdet) und stärkt die Dynamik der Computerspielentwicklung.

Der zweite Teil des Buches beschreibt die Motive und Einschätzungen von Spielenden selbst und macht deutlich, dass Cheating ein überaus heterogenes Phänomen darstellt. Die umfangreiche und faszinierende Analyse fußt auf Interviews mit Spielenden und Akteuren im Feld der Computerspielindustrie, einer teilnehmenden Beobachtung im Online-Rollenspiel Final Fantasy XI, sowie der Analyse wissenschaftlicher Publikationen und Vorträge 
zum Thema Cheating auf Spielentwicklerkonferenzen. Dabei werden sowohl die Logik von Spielregeln beschrieben, als auch verschiedene Arten von Cheats differenziert, Cheater-Typen klassifiziert und mögliche Sanktionen durch die Spielenden skizziert.

Aus Sicht der Spielenden umfasst Cheating nicht nur das Brechen, sondern auch das Dehnen von Spielregeln. Consalvo begreift die Spielenden als aktiv handelnde Subjekte, für die Spielhandlungen mit bestimmten Mehrwerten verbunden sind. Sie zeigt zum einen auf, wie Spieler durch Cheats nicht zwangsläufig zum Nachteil anderer - spezifische Vorteile erlangen und dadurch ihr "gaming capital», ihre Spielerfahrung und den Spaß am Spiel erweitern. Cheating ist aus ihrer Perspektive eine veränderbare Praxis, nicht etwa eine determinierte oder als fixe Identitätseigenschaft. Spielende müssen immer wieder von neuem abwägen, wie sie spielen wollen - ob sie sich Vorteile durch Cheats verschaffen möchten oder Zeit in das Vorankommen im Spiel investieren. Zum anderen beschreibt Consalvo, welche Bewertung mit Blick auf cheatende Spieler herangezogen werden, mit welchen Sanktionen dabei zu rechnen ist und welche Risiken für die Spieler damit verbunden sein können (z.B. das Verspielen von Chancen auf Erfolgserlebnisse, der Verlust von Spielspaß oder gaming capital, verbunden mit Beschädigung der Reputation im Freundeskreis). Die Entscheidungen der Spielenden für bestimmte Spielhandlungen und Cheating werden durch soziale Normen des Umfelds der (Mit-)Spielenden moderiert, aber auch durch Folgen des Handelns (performing) für die Identität des Avatars.

Der dritte Teil des Buches fasst die Position Consalvos zusammen und skizziert einen Ausblick auf noch offene Fragen, die vor allem ethischer Natur sind. Mia Consalvo ist Recht zu geben, wenn sie hier dafür plädiert, dass in der Computerspielforschung die Spielenden selbst, ihre Spielhandlungen und die sie rahmenden Werte und Normen stärker in den Blick genommen werden müssen. Nur so ist es möglich, Erkenntnisse über Computerspiele und ihre Bedeutung für Individuen und unsere Kultur zu erlangen. Wünschenswert wäre es indes, in weiteren Analysen noch stärker Fragen nach der Entwicklung und Durchsetzung von moralischen Ansprüchen zu berücksichtigen. 